\title{
Structure of vacuum deposited SnSe films
}

\author{
T SUBBARAO ${ }^{\dagger}$, B K SAMANTARAY and A K CHAUDHARI \\ Department of Physics, Indian Institute of Technology, Kharagpur 721 302, India \\ ${ }^{+}$Department of Physics, S.K.U.P.G. Centre, Kurnool 518001 , India \\ MS received 15 July 1994; revised 30 October 1994
}

\begin{abstract}
Tin selenide ( $\mathrm{SnSe}_{n}$ ) thin films prepared on mica and glass substrates by vacuum sublimation technique and examined by scanning electron microscopy and transmission electron diffraction techniques showed epitaxial and polycrystalline nature respectively irrespective of substrate temperature. Grain size of the films deposited on glass substrate increased with increase in substrate temperature.
\end{abstract}

Keywords. Electron microscopy; tin selenide films; semiconductors.

\section{Introduction}

SnSe thin films are of great importance because of their use in memory switching devices (Baxter and McLennan 1975). SnSe has a highly anisotropic structure with the tin selenide layers stacked along the b-axis with strong intralayer bonding giving rise to many lattice defects. Using X-ray line profile analysis techniques, Subba Rao et al (1985) studied the different microstructural parameters like crystallite size, rms strain, dislocation density, faulting probability and extent of faults arising due to variability of interlayer spacings in the case of SnSe films deposited on glass substrates. Subba Rao et al (1986) also observed the formation of the high temperature phase (which occurs in bulk SnSe above $807^{\circ} \mathrm{K}$ ) when deposited on glass substrates kept at $473^{\circ} \mathrm{K}$ and above. However, no such $\mathrm{X}$-ray line profile analysis study was undertaken in the case of SnSe films deposited on mica substrates because of overlapping of mica and $\mathrm{SnSe}$ reflections. Hence, an electron microscopic investigation has been undertaken for SnSe thin films vacuum deposited on mica and glass substrates.

\section{Experimental}

Thin films of SnSe were deposited on properly cleaned glass substrates and freshly cleaved (001) planes of mica in a vacuum of $6.67 \times 10^{-3} \mathrm{~Pa}$ with the help of a Hind High vacuum coating unit model $12-\mathrm{A} 4$. During deposition, a substrate temperature of $473^{\circ} \mathrm{K}$ was maintained constant for all the films. The rate of deposition was maintained constant at $30 \mathrm{~nm} / \mathrm{min}$ and for the present investigation, films of thickness $200 \mathrm{~nm}$ have been used. Scanning electron micrographs were obtained by using a Philips model 500 electron microscope at $25 \mathrm{kV}$. Gold films were deposited on the SnSe films by sputtering for improving the contrast. Electron diffraction patterns have been recorded using AEI EM6 transmission electron microscope at $100 \mathrm{kV}$. Samples were prepared following the carbon replica techniques. However the carboncoated specimens had to be made thinner by dissolving the film material in dilute $(10-15 \%)$ nitric acid. Photographs were taken on dia positive plates $\left(0.095 \times 0.08 \mathrm{~m}^{2}\right)$, the exposure time being about $10-20 \mathrm{sec}$. 


\section{Results and discussions}

Two representative scanning electron micrographs for the films deposited on mica and glass substrates are shown in figures $1 \mathrm{a}$ and $\mathrm{c}$ respectively. Figures $1 \mathrm{~b}$ and $\mathrm{d}$ show the electron diffraction patterns from films deposited on mica and glass respectively.

Electron diffraction patterns of films deposited on glass substrates are in the form of continuous rings. This shows that the films are polycrystalline in nature. With increase in the substrate temperature, the rings become more sharp showing the growth of the crystallites and removal of defects. In the case of films deposited on mica substrates the diffraction patterns consist of spots reflecting an epitaxial nature. Higher order of (111) reflections are observed in diffraction patterns from films deposited on mica showing that the films have grown with (111) planes parallel to the substrate. Also the spots became sharper with increase in substrate temperature.
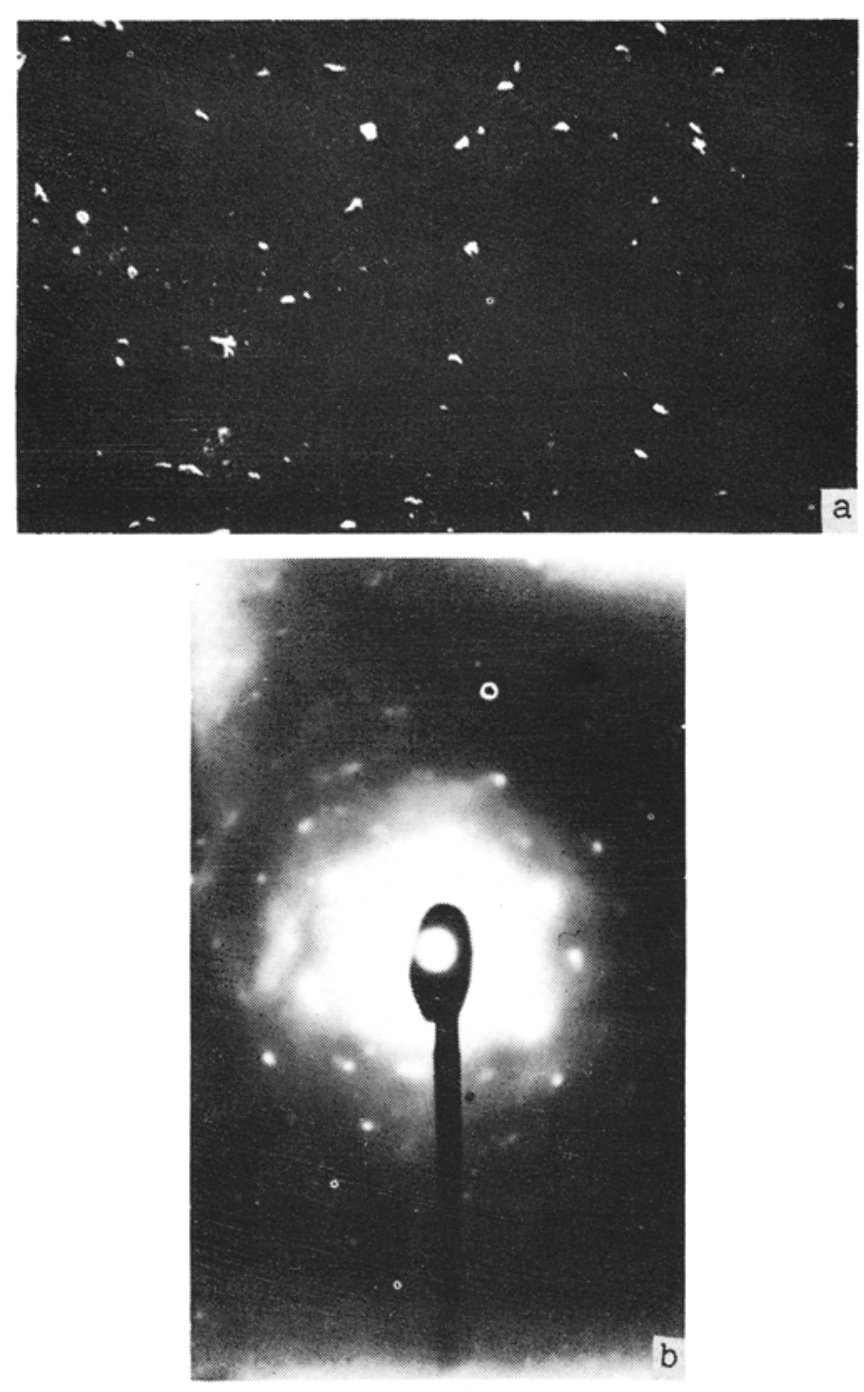

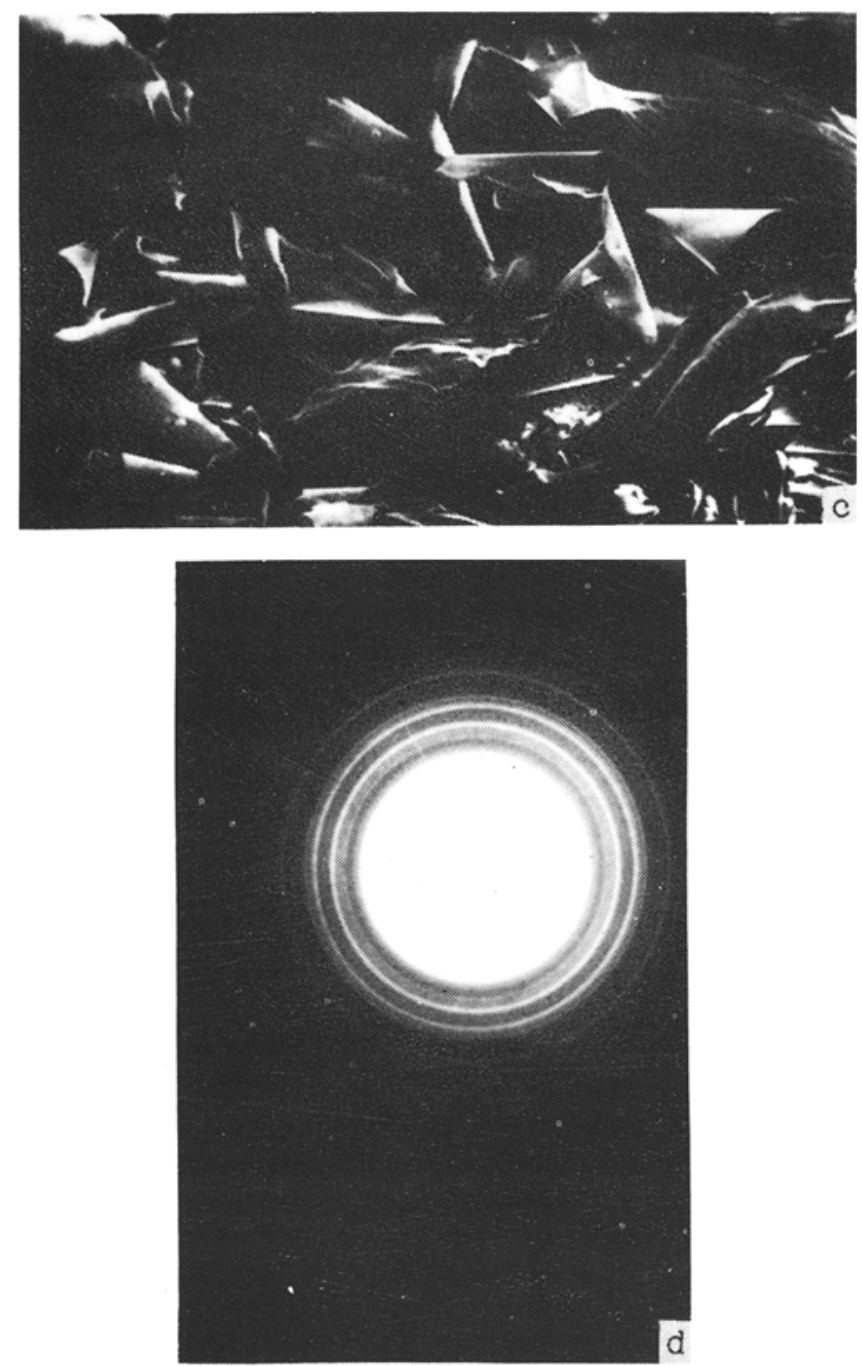

Figure 1. Secondary electron micrographs and electron diffraction pattern, respectively of SnSe films deposited on (a) and (b) mica and (c) and (d) glass substrates at $473^{\circ} \mathrm{K}$.

The SEM patterns from the films deposited on glass substrates show the actual mono crystalline structure clearly. The average size (at $473^{\circ} \mathrm{K}$ substrate temperature) being $80 \mu \mathrm{m}$ along the length and $30 \mu \mathrm{m}$ along the breadth. With increase in substrate temperature there is a slight increase in the crystallite size. The observed size values are much greater than the $\mathrm{X}$-ray crystallite size values reported earlier. This is expected because size values obtained from the $\mathrm{X}$-ray line profile analysis studies correspond to the size of the coherently diffracting domain, whereas the SEM gives the size of the crystallites. One such crystallite may consist of many tiny crystals which are linked by dislocation networks. In SEM patterns of thin films deposited on mica substrates an array of dislocation patterns is observed. This is similar to the observations reported by Amelinck (1964) for $\mathrm{SnS}_{2}$ films in which an extensive network of screw dislocations separated by areas of stacking fault contrast were observed. 


\section{Conclusions}

It was concluded that the SnSe films vacuum-deposited on glass substrates are polycrystalline in nature. Films deposited on mica substrate show epitaxial type spotty patterns with (111) planes parallel to the substrate surface.

\section{References}

Amelinck S 1964 Direct observation of dislocations (New York: Academic Press) p. 271

Baxter C R and McLennan W D 1975 J. Vac. Sci. Technol. 12110

Subba Rao T, Samantaray B K and Chaudhari A K 1985 J. Mater. Sci. Lett. 4743

Subba Rao T, Samantaray B K and Chaudhari A K 1986 Z. Kristallogr. 17537 